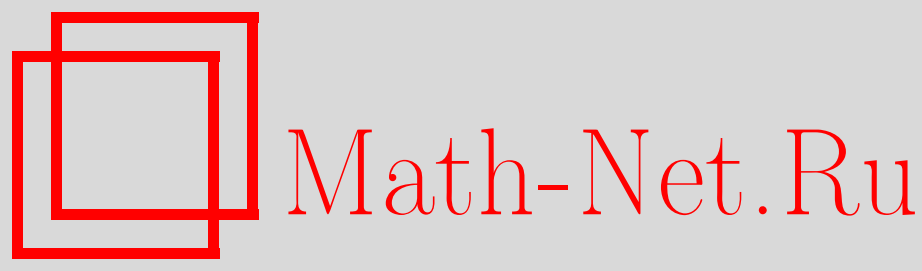

А. Э. Мясникова, Э. Н. Мясников, З. П. Мастропас, Особенности термодинамического потенциала при фазовых переходах второго рода, обусловленных межзонным электрон-фононным взаимодействием, ТМФ, 2008, том 157, номер 2, 273-285

DOI: https://doi.org/10.4213/tmf6279

Использование Общероссийского математического портала Math-Net.Ru подразумевает, что вы прочитали и согласны с пользовательским соглашением http: //www . mathnet.ru/rus/agreement

Параметры загрузки:

IP : 54.197 .217 .227

26 апреля 2023 г., 08:31:08

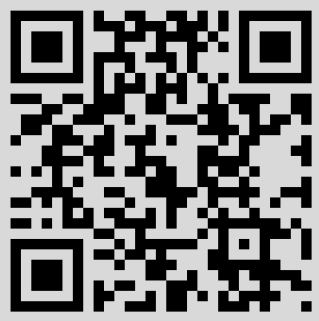


А. Э. Мясникова*, Э.Н. Мясников*, 3. П. Мастропас*

\title{
ОСОБЕННОСТИ ТЕРМОДИНАМИЧЕСКОГО ПОТЕНЦИАЛА ПРИ ФАЗОВЫХ ПЕРЕХОДАХ ВТОРОГО РОДА, ОБУСЛОВЛЕННЫХ МЕЖЗОННЫМ ЭЛЕКТРОН-ФОНОННЫМ ВЗАИМОДЕЙСТВИЕМ
}

\begin{abstract}
Рассмотрены фазовые переходы в кристаллах с сильным межзонным электрон-фононным взаимодействием. Исследование термодинамического потенциала системы проведено методом температурных функций Грина, учитывающим квантовые и тепловые флуктуации. Показано, что в отсутствие стрикции эти фазовые переходы реализуются как череда фазовых переходов второго рода, в каждом из которых термодинамический потенциал имеет логарифмическую особенность, как в модели Онзагера. Это позволяет предположить, что такая особенность характерна для всех фазовых переходов второго рода. Показано, что энергетическую выгодность перехода в упорядоченную фазу обеспечивает взаимодействие электронов с когерентными смещениями ионов по нормальным координатам фононных мод. Вычислено предельное при $T \rightarrow 0 \mathrm{~K}$ значение понижения энергии упорядоченной фазы относительно симметричной.
\end{abstract}

Ключевые слова: термодинамический потенциал, структурная перестройка, электрон-фононное взаимодействие, мода колебания.

1. В многочисленных теоретических и экспериментальных работах по исследованию фазовых переходов второго рода используется запись термодинамического потенциала в виде регулярного разложения по параметру (параметрам) порядка, предложенного впервые Ландау. Особенно широко разложения Ландау по параметрам порядка используются в теории сегнетоэлектрических переходов второго рода [1], в которых обычно параметром порядка является поляризация кристалла. В случае несобственных сегнетоэлектриков [2] в разложениях Ландау параметрами порядка служат некоторые структурные характеристики. Однако в курсе теоретической физики [3] неоднократно подчеркивается, что точка фазового перехода

*Южный федеральный университет, Ростов-на-Дону, Россия.

E-mail: mastrozin@mail.ru 
второго рода должна быть особой точкой термодинамического потенциала, вблизи которой регулярные разложения неприменимы. Границы применимости теории Ландау установил Гинзбург в [4], где фактически доказана возможность применения разложения Ландау во всей области температур, кроме очень узкого участка, не существенного для решения практических задач. Поэтому решение проблемы нахождения характера особенности термодинамического потенциала в точке фазового перехода второго рода на долгое время было сведено к анализу известной модели Изинга [5].

Значительный прогресс в понимании характера указанной особенности связан с теорией критических индексов [6], но степень точности соотношений между ними не позволяет отличить стремление в точке перехода термодинамических величин к постоянному пределу от их логарифмического возрастания. Такую особенность термодинамического потенциала дает единственное известное точное решение задачи о фазовом переходе второго рода [5] в модели Изинга для двумерной дипольной решетки. Ниже будет показано, что логарифмическая расходимость термодинамического потенциала имеет место и при фазовых переходах второго рода, обусловленных сильным межзонным электрон-фононным взаимодействием в кристаллах. Важная роль этого взаимодействия в соединениях с фазовым переходом типа смещения подтверждена в работе [7].

Эти фазовые переходы подробно рассматривались в теории сегнетоэлектрических переходов [8], но достаточно точных вычислений термодинамического потенциала в окрестности точки такого перехода сделать не удавалось. Однако при этом были достигнуты большие успехи в построении квантово-полевой теории таких переходов авторами, работы которых опубликованы в многочисленных статьях в последнее время [9], [10] и достаточно полно отражены в монографии [11]. В этих работах было показано, что ряды теории возмущений для квантовых функций Грина и поляризационного оператора фононов быстро сходятся даже в случае достаточно сильного (для существования фазового перехода) взаимодействия.

2. Используя эти результаты, рассмотрим поправку к термодинамическому потенциалу системы электронов и фононов за счет их межзонного взаимодействия с оператором

$$
H_{\mathrm{int}}=\frac{1}{\sqrt{2 N}} \sum_{\vec{k}, \vec{q}} g_{0}(\vec{q}) \sqrt{\omega_{0}(\vec{q})}\left[a_{2}^{+}(\vec{k}+\vec{q}) a_{1}(\vec{k})+a_{1}^{+}(\vec{k}+\vec{q}) a_{2}(\vec{k})\right]\left(b_{\vec{q}}+b_{-\vec{q}}^{+}\right),
$$

где $b_{\vec{q}}, b_{\vec{q}}^{+}-$операторы уничтожения и рождения фононов с импульсом $\vec{q}$ и частотой $\omega_{0}(\vec{q}), g_{0}(\vec{q})$ - константа их взаимодействия с электронами валентной зоны (индекс 1) и зоны проводимости (индекс 2), поля которых описываются операторами рождения $a_{\sigma}^{+}(\vec{k})$ и уничтожения $a_{\sigma}(\vec{k})$ электронов с одночастичными энергиями $E_{\sigma}(\vec{k}), \sigma=1,2$. Гамильтониан $\widehat{H}_{0}$ свободных полей электронов и фононов имеет вид

$$
\widehat{H}_{0}=\sum_{\vec{k}, \sigma} E_{\sigma}(\vec{k}) a_{\sigma \vec{k}}^{+} a_{\sigma \vec{k}}+\sum_{\vec{q}} \omega(\vec{q}) b_{\vec{q}}^{+} b_{\vec{q}}
$$


В монографии [12] приведена связь между искомой поправкой и температурными функциями Грина фононов в используемой далее системе единиц с $\hbar=1$ :

$$
\Delta \Omega=T \sum_{\vec{q}} \int_{0}^{g_{0}(\vec{q})} \frac{d g}{g} \sum_{\omega_{n}} \frac{\omega_{n}^{2}+\omega_{0}^{2}(\vec{q})}{\omega_{0}^{2}(\vec{q})}\left[D\left(\vec{q}, \omega_{n}\right)-D^{(0)}\left(\vec{q}, \omega_{n}\right)\right]
$$

где $\omega_{n}=2 \pi n T, n$ - целое число. Функция Грина фононов с учетом взаимодействия $D\left(\vec{q}, \omega_{n}\right)$ связана с функцией Грина невзаимодействующих фононов

$$
D^{(0)}\left(\vec{q}, \omega_{n}\right)=\omega_{0}^{2}(\vec{q})\left[\left(i \omega_{n}\right)^{2}-\omega_{0}^{2}(\vec{q})\right]^{-1}
$$

уравнением Дайсона

$$
D\left(\vec{q}, \omega_{n}\right)=D^{(0)}\left(\vec{q}, \omega_{n}\right)+D^{(0)}\left(\vec{q}, \omega_{n}\right) P\left(\vec{q}, \omega_{n}\right) D\left(\vec{q}, \omega_{n}\right)
$$

где $P\left(\vec{q}, \omega_{n}\right)$ - поляризационный оператор фононов. Поэтому выражение (2) удобнее представить в виде

$$
\Delta \Omega=T \sum_{\vec{q}} \int_{0}^{g_{0}(\vec{q})} \frac{d g}{g} \sum_{n} \frac{\omega_{0}^{2}(\vec{q}) P\left(\vec{q}, \omega_{n}\right)}{\left(i \omega_{n}\right)^{2}-\omega_{0}^{2}(\vec{q})\left[1+P\left(\vec{q}, \omega_{n}\right)\right]} .
$$

3. Первые наиболее существенные слагаемые второго порядка по взаимодействию в ряду теории возмущений для поляризационного оператора фононов $P\left(\vec{q}, \omega_{n}\right)$ могут быть представлены двумя диаграммами Фейнмана, отличающимися перестановкой индексов 1 и 2. По правилам соответствия [12] поляризационный оператор в этом приближении имеет аналитический вид

$$
\begin{aligned}
P^{\prime}\left(\vec{q}, \omega_{n}\right)= & \frac{2 T g^{2}}{N} \sum_{\vec{k}, \omega_{m}} \psi\left(i \omega_{n}, i \omega_{m}, \vec{k}, \vec{q}\right) \\
\psi\left(i \omega_{n}, i \omega_{m}, \vec{k}, \vec{q}\right)= & \frac{1}{i \omega_{m}-E_{2}(\vec{k})\left(i\left(\omega_{n}+\omega_{m}\right)-E_{1}(\vec{k}+\vec{q})\right)}+ \\
& \quad+\frac{1}{i \omega_{m}-E_{1}(\vec{k})\left(i\left(\omega_{n}+\omega_{m}\right)-E_{2}(\vec{k}+\vec{q})\right)},
\end{aligned}
$$

где $\omega_{m}=(2 m+1) \pi T, m-$ целое число.

Заметим, что сумма по $\omega_{m}$ в $(5)$ является суммой вычетов по полюсам вспомогательной функции $f(\omega)=-T^{-1}\left\{e^{\omega / T}+1\right\}^{-1}$ подынтегрального выражения в интеграле

$$
\oint_{C_{R} \rightarrow \infty} d \omega f(\omega) \psi\left(i \omega_{n}, \omega, \vec{k}, \vec{q}\right)
$$

по окружности бесконечного радиуса в плоскости комплексного переменного $\omega$.

Поскольку подынтегральное выражение в (7) при $\omega \rightarrow \infty$ стремится к нулю не медленнее, чем $\omega^{-2}$, то сам интеграл (7) равен нулю, и, следовательно, указанная сумма вычетов должна равняться сумме вычетов в полюсах функции $\psi\left(i \omega_{n}, \omega, \vec{k}, \vec{q}\right)$ на плоскости комплексного переменного $\omega$, взятой с обратным знаком. 
Таким образом, бесконечную сумму по $\omega_{m}$ в (5) можно заменить выражением

$$
\begin{gathered}
-\frac{f\left(E_{2}(\vec{k})\right)}{i \omega_{n}+E_{2}(\vec{k})-E_{1}(\vec{k}+\vec{q})}+\frac{f\left(E_{1}(\vec{k}+\vec{q})-i \omega_{n}\right.}{i \omega_{n}+E_{2}(\vec{k})-E_{1}(\vec{k}+\vec{q})}- \\
-\frac{f\left(E_{1}(\vec{k})\right)}{i \omega_{n}+E_{1}(\vec{k})-E_{2}(\vec{k}+\vec{q})}+\frac{f\left(E_{2}(\vec{k}+\vec{q})-i \omega_{n}\right)}{i \omega_{n}+E_{1}(\vec{k})-E_{2}(\vec{k}+\vec{q})} .
\end{gathered}
$$

Для простоты предположим, что рассматриваемый полупроводник имеет широкую запрещенную зону (с шириной, значительно большей величины температуры), а зонные энергии отсчитываются от уровня химического потенциала, расположенного в середине запрещенной зоны. В таком случае выражение (8) сильно упрощается:

$$
\frac{1}{T}\left\{\frac{1}{E_{1}(\vec{k}+\vec{q})-i \omega_{n}-E_{2}(\vec{k})}+\frac{1}{i \omega_{n}+E_{1}(\vec{k})-E_{2}(\vec{k}+\vec{q})}\right\} .
$$

Поэтому для кристаллов с центром инверсии в высокосимметричной фазе выражение (5) принимает вид

$$
P^{\prime}\left(\vec{q}, i \omega_{n}\right)=\frac{4 g^{2} \bar{\Delta}(\vec{q})}{\left(i \omega_{n}\right)^{2}-\bar{\Delta}^{2}(\vec{q})}
$$

в котором величину $\bar{\Delta}(\vec{q})$, полученную в результате суммирования по $\vec{k}$ и деления на $N$, назовем средним межзонным расстоянием между подуровнями двух электронных зон, отличающимися по импульсу на $\vec{q}$.

4. Подставляя (10) в (4), получаем

$$
\begin{aligned}
\Delta \Omega=- & T \sum_{\vec{q}} \int_{0}^{g(q)} d g 4 g \omega_{0}^{2}(\vec{q}) \bar{\Delta}(\vec{q}) \times \\
& \times \sum_{n} \frac{1}{\left[\left(i \omega_{n}\right)^{2}-\bar{\Delta}^{2}(\vec{q})\right]\left[\left(i \omega_{n}\right)^{2}-\omega_{0}^{2}(\vec{q})\right]-4 g^{2} \omega_{0}^{2}(\vec{q}) \bar{\Delta}(\vec{q})} .
\end{aligned}
$$

Вычисление входящей в (11) суммы по $n$ производится аналогично суммированию в (5) с помощью вспомогательной функции $\varphi(\omega)=T^{-1}\left\{e^{\omega / T}-1\right\}^{-1}$. Результат определяется положением полюсов функции

$$
\psi^{\prime}(\omega, \vec{q})=\frac{1}{\left[\omega^{2}-\bar{\Delta}^{2}(\vec{q})\right]\left[\omega^{2}-\omega_{0}^{2}(\vec{q})\right]-4 g^{2} \omega_{0}^{2}(\vec{q}) \bar{\Delta}(\vec{q})} .
$$

Если запрещенная зона настолько широка, что $\bar{\Delta}(\vec{q}) \gg \omega_{0}(\vec{q})$, то положение четырех ее полюсов определяется равенствами:

$$
\begin{aligned}
& \omega^{2}=\omega_{1}^{2}(\vec{q}) \approx \bar{\Delta}^{2}(\vec{q})+\frac{4 g^{2} \omega_{0}^{2}(\vec{q})}{\bar{\Delta}(\vec{q})} \approx \bar{\Delta}^{2}(\vec{q}), \\
& \omega^{2}=\omega_{2}^{2}(\vec{q}) \approx \omega_{0}^{2}(\vec{q})\left[1-\frac{4 g^{2}}{\bar{\Delta}(\vec{q})}\right],
\end{aligned}
$$


а величина (11) принимает вид

$$
\Delta \Omega=-\sum_{\vec{q}} \int_{0}^{g_{0}(\vec{q})} 2 g d g\left[2 n\left(\omega_{2}(\vec{q}), T\right)+1\right] \frac{\omega_{0}^{2}(\vec{q})}{\bar{\Delta}(\vec{q}) \omega_{2}(\vec{q})},
$$

где $n\left(\omega_{2}(\vec{q}), T\right)=T \varphi\left(\omega_{2}(\vec{q})\right)=\exp \left(\omega_{2}(\vec{q}) / T-1\right)^{-1}$. Второе равенство в (12) показывает, что в используемом приближении квадрат частоты фононов, перенормированный взаимодействием, может оказаться отрицательным. Это свидетельствует о неустойчивости высокосимметричной фазы при $4 g_{0}^{2}(\vec{q})>\bar{\Delta}(\vec{q})$, т.е. при достаточно сильном взаимодействии. Поскольку величина $\bar{\Delta}(\vec{q})$ практически для всех полупроводников порядка 1 эВ, то следует ожидать, что величина $g_{0}^{2}(\vec{q})$ в некоторых веществах и для фононных мод в небольшой окрестности некоторой точки в первой зоне Бриллюэна может равняться и даже немного превосходить величину $\bar{\Delta}(\vec{q}) / 4$. В таком случае под влиянием электрон-фононного взаимодействия возможны фазовые переходы в упорядоченную фазу. И в этом случае оба слагаемых в выражении (12) для $\omega_{2}^{2}(\vec{q})$ оказываются примерно одного порядка, а следовательно, необходимо учитывать поправку четвертого порядка к поляризационному оператору. Согласно [11] с учетом этой поправки

$$
\omega_{2}^{2}(\vec{q})=\omega_{0}^{2}(\vec{q})\left[1-\frac{4 g^{2}}{\bar{\Delta}(\vec{q})}+\frac{48 g^{4} T}{4 \bar{\Delta}^{3}(\vec{q})}\right] .
$$

5. В новом приближении частота $\omega_{2}(\vec{q})$ обращается в нуль при

$$
T=T_{\mathrm{c}}(\vec{q})=\left[4 g_{0}^{2}(\vec{q})-\bar{\Delta}(\vec{q})\right] \frac{\bar{\Delta}^{2}(\vec{q})}{24 g_{0}^{4}(\vec{q})},
$$

а если вместо переменной $g$ использовать $g_{0}(\vec{q})$, то при $T>T_{\mathrm{c}}(\vec{q})$ она положительна. Но в (13) частота (14) входит в подынтегральное выражение. Сложный интеграл по $g$ в (13) можно значительно упростить. Предположим, что только для одной моды с волновым вектором $\vec{q}_{0}$ величина в правой части равенства (15) положительна, и рассмотрим слагаемое в $(13)$, соответствующее $\vec{q}_{0}$. Пусть $T_{\mathrm{c}}\left(\vec{q}_{0}\right)$ значительно больше частоты $\omega_{2}\left(\vec{q}_{0}\right)$, вычисленной по формуле $(14)$ с константой связи $g_{0}\left(\vec{q}_{0}\right)$. Тогда при $T>T_{\mathrm{c}}\left(\vec{q}_{0}\right)$

$$
2 n\left(\omega_{2}\left(\vec{q}_{0}\right), T\right)+1 \approx \frac{2 T}{\omega_{2}\left(\vec{q}_{0}\right)} .
$$

В этом случае интеграл по $g$ в соответствующем слагаемом в (13) легко вычисляется. В результате получаем

$$
\Delta \Omega\left(\vec{q}_{0}\right)=-\frac{T}{2} \ln \left\{\frac{\alpha_{-}}{\alpha_{+}} \frac{\beta_{-}}{\beta_{+}}\right\},
$$

где $\alpha_{ \pm}=12 T g_{0}^{2}\left(\vec{q}_{0}\right)\left[\bar{\Delta}^{2}\left(\vec{q}_{0}\right)\right]^{-1}-\beta_{ \pm}, \beta_{ \pm}=1 \pm \sqrt{1-6 T / \bar{\Delta}\left(\vec{q}_{0}\right)}$. Аргумент логарифма в (17) обращается в нуль при $T=T_{\mathrm{c}}\left(\vec{q}_{0}\right)(15)$. При этой же температуре обращается в нуль и частота $\omega_{2}\left(\vec{q}_{0}\right)$, если в $(14)$ заменить переменную $g$ на реальную константу связи $g_{0}\left(\vec{q}_{0}\right)$. Это означает, что при температуре $T_{\mathrm{c}}\left(\vec{q}_{0}\right)$ в системе произойдет фазовый переход второго рода с обращением в нуль частоты “мягкой" моды [11] и появлением логарифмически расходящегося слагаемого в выражении для термодинамического потенциала. 
6. Заметим, что выражение (15) после разрешения относительно $\bar{\Delta} / 4 g^{2}$ приводит при условии $6 T_{\mathrm{c}} \ll \bar{\Delta}$ к соотношению

$$
\frac{\bar{\Delta}}{4 g^{2}}=1-\frac{3 T_{\mathrm{c}}}{2 \bar{\Delta}} .
$$

У сегнетоэлектриков $\mathrm{BaTiO}_{3}$ и $\mathrm{SbSI}$ отношение $T_{\mathrm{c}} / \bar{\Delta}$ порядка $10^{-2}$, и, следовательно, в этих веществах величина $4 g^{2}$ с точностью до процентов равна $\bar{\Delta}$.

Представляет интерес исследовать поведение этого расходящегося слагаемого в выражении для $\Delta \Omega$ при температурах, близких к $T_{\mathrm{c}}\left(q_{0}\right)$. Заменяя в выражении (17) $T$ на $T_{\mathrm{c}}+t, t=T-T_{\mathrm{c}}$, и учитывая соотношение $(15)$, получаем при $t \ll T_{\mathrm{c}}$

$$
\Delta \Omega\left(q_{0}\right)=-\frac{T_{\mathrm{c}}}{2} \ln \left\{3 \frac{t}{\bar{\Delta}}\left[1-\frac{1}{2}\left(\frac{4 g_{0}^{2}}{\bar{\Delta}}\right)^{2}\right]\right\} .
$$

Если $t \ll T_{\mathrm{c}} \ll \bar{\Delta} / 6$, то соотношение $(19)$ принимает простой вид:

$$
\Delta \Omega\left(q_{0}\right)=-\frac{T_{\mathrm{c}}}{2} \ln \frac{3 t}{2 \bar{\Delta}} .
$$

Логарифм в (19) и (20) отрицателен, поэтому при $T \rightarrow T_{\mathrm{c}} \Delta \Omega\left(q_{0}\right)$ возрастает. Это возрастание заканчивается в результате обращения в нуль частоты "мягкой моды" [11] и появления когерентных смещений ионов (т.е. квантовых средних смещений ионов). При $q_{0}=0$ эти смещения однородны по объему кристалла.

Если взаимодействие слабое, так что $\bar{\Delta}>4 g^{2}$ для всех мод, то в выражении для $\Delta \Omega$ не будет расходящихся слагаемых, а их величину легко оценить, считая, что

$$
\omega_{2}^{2}(\vec{q})=\omega_{0}^{2}(\vec{q})\left[1-4 g^{2}(\vec{q}) \bar{\Delta}^{-1}(\vec{q})\right]
$$

Поскольку число этих слагаемых в сумме (13) даже для кристалла миллиметровых размеров огромно (порядка $10^{20}$ ), одно сингулярное слагаемое типа (19) или (20) будет заметным на фоне огромного числа несингулярных слагаемых лишь при ничтожно малом отношении $t / \bar{\Delta}$, например при $t / \bar{\Delta} \approx e^{-10^{15}}$. Естественно, что, заменив сумму в (13) (которая является суммой из-за финитности движения рассматриваемых квазичастиц) интегралом, мы получим нерасходящийся термодинамический потенциал, который можно использовать при температурах, практически сколь угодно близких к $T_{\mathrm{c}}$, и, следовательно, при этих температурах возможны регулярные разложения термодинамического потенциала. Совершенно аналогичная ситуация имеет место в решении Онзагера [5] для решетки Изинга. В этом случае термодинамический потенциал представляет собой сумму логарифмических слагаемых, в одном из которых аргумент обращается в нуль при $T \rightarrow T_{\mathrm{c}}$. Поскольку остальные слагаемые в сумме регулярны при $T \rightarrow T_{\mathrm{c}}$, то и для всей суммы $T_{\mathrm{c}}$ является точкой разрыва, как это и требуется в соответствии с [5]. Не составляет большого труда доказать, что расходящееся слагаемое в этой сумме при $t=T-T_{\mathrm{c}} \rightarrow 0$ ведет себя как $\ln \left(t / T_{\mathrm{c}}\right)$. Действительно, расходящееся слагаемое в термодинамическом потенциале решетки Изинга можно представить в виде $\ln \left(\operatorname{th}\left(I / T_{\mathrm{c}}\right)-\operatorname{th}(I / T)\right)$, где $I$ - 
величина энергии взаимодействия соседних диполей в решетке Изинга. При очень малых $t=T-T_{\mathrm{c}}$

$$
\ln \left(\operatorname{th} \frac{I}{T_{\mathrm{c}}}-\operatorname{th} \frac{I}{T}\right) \approx \ln \frac{t}{T_{\mathrm{c}}}+\ln \left(\frac{I}{T_{\mathrm{c}}}\left(1+\operatorname{th}^{2} \frac{I}{T_{\mathrm{c}}}\right)\right) .
$$

Таким образом, термодинамический потенциал в решении Онзагера имеет такую же логарифмическую расходимость, как и в рассмотренном нами выше случае (21). Это позволяет надеяться, что такая особенность термодинамического потенциала характерна для любого фазового перехода второго рода. Естественно, что при замене суммы, представляющей термодинамический потенциал интегралом, логарифмическая расходимость исчезает. В решении Онзагера зависимость слагаемых от индексов суммирования такова, что интегрирование приводит к появлению особого слагаемого $T_{\mathrm{c}} t^{2} \ln t$, которое не расходится при $t \rightarrow 0$. Оно и определяет температурную зависимость термодинамических характеристик вдали от очень узкой окрестности $T_{\mathrm{c}}$, в которой вклад расходящегося слагаемого в термодинамический потенциал существенен и не пропадает на фоне суммы огромного числа регулярных слагаемых.

Таким образом, фазовые переходы второго рода, обусловленные межзонным электрон-фононным взаимодействием, возникают в результате логарифмического возрастания термодинамического потенциала в малой окрестности точки перехода $T_{\mathrm{c}}$. Это приводит к термодинамической выгодности новой фазы с отличными от нуля смещениями положения равновесия ионов. Можно показать, что именно взаимодействие электронов с этими смещениями понижает энергию кристалла.

7. Применим к операторам рождения и уничтожения фононов, входящим в гамильтониан $\widehat{H}_{0}+\widehat{H}_{\mathrm{int}}(1)$, унитарное преобразование, позволяющее выделить в отдельную классическую переменную $d_{\vec{k}}$ среднее значение оператора уничтожения фонона $\vec{k}$-й моды, т.е. сумму (с соответствующими коэффициентами) средних значений координаты $q$ и импульса $p \vec{k}$-й гармоники колебаний решетки [13]:

$$
U(d)=\prod_{\vec{k}} \exp \left[d_{\vec{k}} b_{\vec{k}^{\prime}}^{+}-d_{\vec{k}}^{*} b_{\vec{k}^{\prime}}\right]=\prod_{\vec{k}} \exp \left[\frac{i}{\hbar}\left(p_{\vec{k}} \widehat{Q}_{\vec{k}^{\prime}}-q_{\vec{k}} \widehat{P}_{\vec{k}^{\prime}}\right)\right] .
$$

При этом преобразовании новый оператор $b_{\vec{k}}^{\prime}=U b_{\vec{k}} U^{-1}=b_{\vec{k}}-d_{\vec{k}}$, а $b_{\vec{k}}^{\prime+}=U b_{\vec{k}}^{+} U^{-1}=$ $b_{\vec{k}}^{+}-d_{\vec{k}}^{*}$, поэтому среднее значение нового оператора $b_{\vec{k}}^{\prime}$ будет равно нулю. Новый гамильтониан $\widehat{H}^{\prime}$ после такого унитарного преобразования имеет вид

$$
\begin{aligned}
\widehat{H}^{\prime} \equiv U \widehat{H} U^{-1}= & \sum_{\vec{k}, \sigma=1}^{2} E_{\sigma}(\vec{k}) a_{\sigma \vec{k}}^{+} a_{\sigma \vec{k}}+\sum_{\vec{q}} \omega(\vec{q}) b_{\vec{q}}^{\prime+} b_{\vec{q}}^{\prime}+ \\
& +\sum_{\substack{\vec{k}, \vec{q} \\
\sigma \neq \sigma^{\prime}=1}}^{2} \sqrt{\frac{\omega(\vec{q})}{2 N}} g_{0}(\vec{q}) a_{\vec{k} \sigma}^{+} a_{\vec{k}-\vec{q}, \sigma^{\prime}}\left(b_{\vec{q}}^{\prime}+b_{\vec{q}}^{\prime+}\right) .
\end{aligned}
$$


Пользуясь указанными выше правилами преобразования для операторов $b_{\vec{k}}^{\prime}$ и $b_{\vec{k}}^{\prime}$, этот гамильтониан можно представить в виде

$$
\begin{aligned}
& \widehat{H}^{\prime}=\sum_{\vec{k}, \sigma=1}^{2} E_{\sigma}(\vec{k}) a_{\sigma \vec{k}}^{+} a_{\sigma \vec{k}}+\sum_{\vec{q}} \omega(\vec{q}) b_{\vec{q}}^{+} b_{\vec{q}}+ \\
& \quad+\sum_{\substack{\vec{k}, \vec{q} \\
\sigma \neq \sigma^{\prime}=1}}^{2} \sqrt{\frac{\omega(\vec{q})}{2 N}} g_{0}(\vec{q}) a_{\vec{k} \sigma}^{+} a_{\vec{k} \sigma}\left(b_{\vec{q}}+b_{-\vec{q}}^{+}\right)+\widehat{H}^{\prime \prime}, \\
& \widehat{H}^{\prime \prime}=\sum_{\vec{q}} \omega(\vec{q})\left(d_{\vec{q}}^{*} d_{\vec{q}}-b_{\vec{q}}^{*} d_{\vec{q}}-d_{\vec{q}}^{*} b_{\vec{q}}\right)-\sum_{\substack{\vec{q}, \vec{k} \\
\sigma \neq \sigma^{\prime}}} \sqrt{\frac{\omega(\vec{q})}{2 N}} g_{0}(\vec{q}) a_{\vec{k} \sigma}^{+} a_{\vec{k}-\vec{q}, \sigma^{\prime}}\left(d_{\vec{q}}+d_{-\vec{q}}^{*}\right) .
\end{aligned}
$$

Найдем сначала вариационным методом основное состояние системы, в котором среднее значение гамильтониана $\widehat{H}=\widehat{H}^{\prime}-\widehat{H}^{\prime \prime}$ должно быть минимальным. В качестве варьируемого вектора основного состояния используем вектор

$$
|d, \psi\rangle \equiv \psi \exp \left[\sum_{\vec{q}} \exp \left(d_{\vec{q}} b_{\vec{q}}^{+}-d_{\vec{q}}^{*} b_{\vec{q}}\right)\right]|0\rangle=\psi U(d)|0\rangle,
$$

где $|0\rangle$ - вектор состояния фононного поля без фононов и без деформации вакуума, а варьируемые параметры $d_{\vec{q}}$ совпадают с параметрами, содержащимися в выражении (22). Волновую функцию $\psi$ электронной подсистемы в представлении чисел заполнения с ее варьируемыми параметрами определим позже.

8. Найдем среднее значение оператора $\widehat{H}=\widehat{H}^{\prime}-\widehat{H}^{\prime \prime}$ в состоянии $|d, \psi\rangle$. Имеем

$$
\left\langle d, \psi\left|\widehat{H}^{\prime}\right| d, \psi\right\rangle=\sum_{\vec{k}, \sigma=1}^{2} E_{\sigma}(\vec{k})\left\langle\psi\left|a_{\sigma \vec{k}}^{+} a_{\sigma \vec{k}}\right| \psi\right\rangle
$$

так как $\left\langle 0\left|b_{\vec{q}}^{\prime+} b_{\vec{q}}^{\prime}\right| 0\right\rangle=0$ и $\left\langle 0\left|b_{\vec{q}}^{\prime}\right| 0\right\rangle=\left\langle 0\left|b_{\vec{q}}^{\prime}{ }^{+}\right| 0\right\rangle=0$. Аналогично получаем

$$
\left\langle d, \psi\left|\widehat{H}^{\prime \prime}\right| d, \psi\right\rangle=-\sum_{\vec{q}}\left[\omega(\vec{q}) d_{\vec{q}}^{*} d_{\vec{q}}+\sum_{\vec{k}, \sigma \neq \sigma^{\prime}} \sqrt{\frac{\omega(\vec{q})}{2 N}} g_{0}(\vec{q})\left\langle\psi\left|a_{\vec{k} \sigma}^{+} a_{\vec{k}-\vec{q}, \sigma^{\prime}}\right| \psi\right\rangle\left(d_{\vec{q}}+d_{-\vec{q}}^{+}\right)\right],
$$

поскольку $\left\langle d, \psi\left|b_{\vec{q}}\right| d, \psi\right\rangle=d_{\vec{q}},\left\langle d, \psi\left|b_{\vec{q}}^{+}\right| d, \psi\right\rangle=d_{\vec{q}}^{*}$. Таким образом, имеем

$$
\begin{aligned}
\langle d, \psi|\widehat{H}| d, \psi\rangle= & \sum_{\vec{k}, \sigma=1}^{2} E_{\sigma}(\vec{k})\left\langle\psi\left|a_{\sigma \vec{k}}^{+} a_{\sigma \vec{k}}\right| \psi\right\rangle+\sum_{\vec{q}}\left[\omega(\vec{q}) d_{\vec{q}}^{*} d_{\vec{q}}+\right. \\
& \left.\quad+\sum_{\vec{k}, \sigma \neq \sigma^{\prime}} \sqrt{\frac{\omega(\vec{q})}{2 N}} g_{0}(\vec{q})\left\langle\psi\left|a_{\vec{k} \sigma}^{+} a_{\vec{k}-\vec{q}, \sigma^{\prime}}\right| \psi\right\rangle\left(d_{\vec{q}}+d_{-\vec{q}}^{*}\right)\right] .
\end{aligned}
$$

Величина среднего смещения $\vec{q}$-й моды колебания

$$
\bar{u}_{\vec{q}}=\sqrt{\frac{M \omega(\vec{q})}{2}}\left\langle\psi\left|b_{\vec{q}}+b_{\vec{q}}^{+}\right| \psi\right\rangle=\sqrt{\frac{M \omega(\vec{q})}{2}}\left(d_{\vec{q}}+d_{-\vec{q}}^{*}\right)
$$


должна быть вещественной, следовательно, $d_{\vec{q}}=d_{-\vec{q}}$. Делая подстановку $d_{\vec{q}}=$ $\left|d_{\vec{q}}\right| e^{i \varphi_{\vec{q}}}$ и приравнивая нулю производную по $\left|d_{\vec{q}}\right|$ функции $(26)$, найдем экстремум по $\left|d_{\vec{q}}\right|$ :

$$
\begin{aligned}
\langle d, \psi|\widehat{H}| d, \psi\rangle & =\sum_{\vec{k}, \sigma=1}^{2} E_{\sigma}(\vec{k})\left\langle\psi\left|a_{\sigma \vec{k}}^{+} a_{\sigma \vec{k}}\right| \psi\right\rangle-\sum_{\vec{q}} \omega(\vec{q})\left|d_{\vec{q}}\right|^{2}, \\
\left|d_{\vec{q}}\right| & =-\frac{\cos \varphi(\vec{q})}{\sqrt{2 N \omega(\vec{q})}} \sum_{\vec{k}, \sigma \neq \sigma^{\prime}} g_{0}(\vec{q})\left\langle\psi\left|a_{\sigma \vec{k}}^{+} a_{\sigma^{\prime}, \vec{k}-\vec{q}}\right| \psi\right\rangle .
\end{aligned}
$$

Таким образом, при достаточно сильном электрон-фононном взаимодействии энергия деформации решетки оказывается в два раза меньше отрицательной энергии взаимодействия электронов с деформацией решетки.

Поскольку оказалось (см. (27)), что энергия взаимодействия отрицательна и превосходит собственную энергию деформации каждой гармоники, а $\left|d_{\vec{q}}\right|$ должно быть положительным, то минимуму среднего значения гамильтониана $\widehat{H}$ по $\varphi_{\vec{q}}$ будет соответствовать значение $\cos \varphi_{\vec{q}}=-1$, т.е. величина параметра $d_{\vec{q}}$ вещественна и, следовательно, межзонное взаимодействие только смещает положения равновесия ионов.

Таким образом, среди всех возможных деформаций минимуму полной энергии соответствует деформация с компонентами $\left|d_{\vec{q}}\right|$ из (27).

9. Волновые функции состояния электронной подсистемы при учете межзонного электрон-фононного взаимодействия должны быть суперпозицией блоховских волновых функций из обеих зон. Оператор электрон-фононного взаимодействия переводит состояние электрона $(\sigma, \vec{k})$ в состояние $\left(\sigma^{\prime}, \vec{k}-\vec{q}\right)$, поэтому в такой двухзонной модели в качестве одночастичных волновых функций электронов можно использовать $\varphi_{1 \vec{k}}$ для валентной зоны $(\sigma=1)$ и $\varphi_{2 \vec{k}}$ для зоны проводимости $(\sigma=2)$ :

$$
\begin{aligned}
& \varphi_{1 \vec{k}}=\frac{1}{\sqrt{V}}\left[C_{11}(\vec{k}) u_{1 \vec{k}}(\vec{r}) e^{i \vec{k} \vec{r}}+\sum_{\vec{q}} C_{12}(\vec{k}, \vec{q}) u_{2 \vec{k}-\vec{q}}(\vec{r}) e^{i(\vec{k}-\vec{q}) \vec{r}}\right], \\
& \varphi_{2 \vec{k}}=\sqrt{V}\left[C_{22}(\vec{k}) u_{2 \vec{k}}(\vec{r}) e^{i \vec{k} \vec{r}}+\sum_{\vec{q}} C_{21}(\vec{k}, \vec{q}) u_{1 \vec{k}-\vec{q}}(\vec{r}) e^{i(\vec{k}-\vec{q}) \vec{r}}\right],
\end{aligned}
$$

где блоховские амплитуды $u_{\sigma \vec{k}}(\vec{r})$ являются периодическими функциями переменной $\vec{r}$ с периодом решетки кристалла в неупорядоченной фазе, $V$ - объем кристалла. Блоховские волновые функции в соотношениях (28) считаются ортонормированными, поэтому на вариационные параметры $C_{11}, C_{12}, C_{22}, C_{21}$ условиями нормировки налагаются следующие ограничения:

$$
\left|C_{11}(\vec{k})\right|^{2}+\sum_{\vec{q}}\left|C_{12}(\vec{k}, \vec{q})\right|^{2}=1, \quad\left|C_{22}(\vec{k})\right|^{2}+\sum_{\vec{q}}\left|C_{21}(\vec{k}, \vec{q})\right|^{2}=1 .
$$

Если считать, что электроны в основном состоянии находятся только в нижней зоне в состояниях с волновыми функциями $\varphi_{1 \vec{k}}$, а их спины, для простоты, имеют одну, одинаковую для обеих зон ориентацию, то многочастичную антисимметричную по 
перестановкам переменных волновую функцию $\psi$ системы электронов можно представить в виде определителя из функций $\varphi_{2 \vec{k}}$ с числами заполнения $n_{1 \vec{k}}=1, n_{2 \vec{k}}=0$.

Соотношения (28) позволяют построить новые операторы рождения $\alpha_{\sigma \vec{k}}^{+}$и уничтожения $\alpha_{\sigma \vec{k}}$ электронов в состояниях с одночастичными волновыми функциями $\varphi_{\sigma \vec{k}}(\vec{r})$ как линейные комбинации операторов $\alpha_{\sigma \vec{k}}^{+}, \alpha_{\sigma \vec{k}}$. Для этого приравняем разложения оператора поля электронов $\Psi(\vec{r})$ по блоховским волновым функциям и по функциям $\varphi_{\sigma \vec{k}}(\vec{r})$ :

$$
\frac{1}{\sqrt{V}} \sum_{\sigma, \vec{k}} a_{\sigma \vec{k}} u_{\sigma \vec{k}} e^{i \vec{k} \vec{r}}=\sum_{\sigma, \vec{k}} a_{\sigma \vec{k}} \varphi_{\sigma \vec{k}}(\vec{r}) .
$$

Обычно [11] в теории фазовых переходов под влиянием межзонного электрон-фононного взаимодействия рассматривается простейший случай, в котором в (28) в сумме по $\vec{q}$ учитывается только одно слагаемое, соответствующее $\vec{q}=0$. Таким образом, предполагается, что решетка однородно деформируется по нормальной координате, соответствующей моде с $\vec{q}=0$. Это предположение довольно грубое, но оно позволяет значительно упростить вычисления. Далее мы также будем пользоваться таким предположением. В этом случае функции $\varphi_{1 \vec{k}}$ и $\varphi_{2 \vec{k}}$ оказываются ортонормированными при $\left|C_{11}(\vec{k})\right|^{2}+\left|C_{12}(\vec{k})\right|^{2}=1$. Равенство (30) приводит к соотношениям

$$
a_{1 \vec{k}}=\alpha_{1 \vec{k}} C_{11}(\vec{k})+\alpha_{2 \vec{k}} C_{21}(\vec{k}), \quad a_{2 \vec{k}}=\alpha_{2 \vec{k}} C_{22}(\vec{k})+\alpha_{1 \vec{k}} C_{12}(\vec{k}) .
$$

Подставляя (31) в (27), усредняя по указанной выше многочастичной волновой функции с числами заполнения $n_{1 \vec{k}}=1, n_{2 \vec{k}}=0$ и учитывая, что $a_{2 \vec{q}}|d, \psi\rangle=0$, получаем

$$
\begin{aligned}
\langle d, \psi|\widehat{H}| d, \psi\rangle & =\sum_{\vec{k}}\left(E_{1}(\vec{k})+\Delta(\vec{k})\left|C_{12}(\vec{k})\right|^{2}\right)-\sum_{\vec{q}} \omega(\vec{q})\left|d_{\vec{q}}\right|^{2}, \\
\left|d_{\vec{q}}\right| & =\frac{1}{\sqrt{2 N \omega(\vec{q})}} g_{0}(\vec{q}) \sum_{\vec{k}}\left(C_{11}^{*}(\vec{k}) C_{12}(\vec{k})+C_{11}(\vec{k}) C_{12}^{*}(\vec{k})\right),
\end{aligned}
$$

где $\Delta(\vec{k})=E_{2}(\vec{k})-E_{1}(\vec{k})$.

10. В формулах (32) коэффициенты $C_{11}$ и $C_{12}$ зависят только от $\vec{k}$. Поскольку вершинная часть $g_{0}$ не зависит от волновых векторов $\vec{k}$, можно полагать, что, вообще говоря, комплексные коэффициенты $C_{11}$ и $C_{12}$ также не зависят от $\vec{k}$ или зависят от волнового вектора $\vec{k}$ только через свою фазу $f$ :

$$
C_{11}(\vec{k})=\left|C_{11}\right| e^{i f_{1}(\vec{k})}, \quad C_{12}(\vec{k})=\left|C_{12}\right| e^{i f_{2}(\vec{k})} .
$$

Поэтому в выражении для $\left|d_{\vec{q}}\right|$, вынося модули $\left|C_{11}\right|,\left|C_{12}\right|$ за знак суммирования по $\vec{k}$, получаем

$$
\frac{g_{0}(\vec{q})}{N} \sum_{\vec{k}}\left[e^{i\left(f_{2}-f_{1}\right)}+e^{i\left(f_{1}-f_{2}\right)}\right]=\frac{2 g_{0}(\vec{q})}{N} \sum_{\vec{k}} \cos \left(f_{2}(\vec{k})-f_{1}(\vec{k})\right) .
$$


Минимуму энергии будет соответствовать максимальное значение суммы (34). Поэтому варьирование фаз $f_{2}$ и $f_{1}$ приводит к равенству $f_{2}(\vec{k})=f_{1}(\vec{k})$. Учитывая $(34)$, получим более простые соотношения

$$
\begin{gathered}
\left|d_{\vec{q}}\right|=2 \sqrt{N} \frac{g_{0}(\vec{q})}{\sqrt{2 \omega(\vec{q})}}\left|C_{11}\right|\left|C_{12}\right|, \\
\langle d, \psi|\widehat{H}| d, \psi\rangle=\sum_{\vec{k}} E_{1}(\vec{k})+\left(1-\left|C_{11}\right|^{2}\right) \sum_{\vec{k}} \Delta(\vec{k})-\sum_{\vec{q}} 2 N g_{0}(\vec{q})\left|C_{11}\right|^{2}\left|C_{12}\right|^{2} .
\end{gathered}
$$

Теперь найдем минимум энергии (32) по параметрам $\left|C_{11}\right|$ и $\left|C_{12}\right|$. Поскольку эти параметры связаны условием нормировки, то фактически мы имеем один варьируемый параметр.

Исследуем случай однородной деформации с $g_{0}(\vec{q})=g_{0} \delta_{\vec{q} 0}$, соответствующий известной межзонной теории сегнетоэлектрических фазовых переходов, в которой рассматривается поведение одной (“мягкой”) моды колебаний под влиянием взаимодействия с электронами. Используя в качестве вариационного один коэффициент $\left|C_{12}\right|$, на основе (32) получаем

$$
\left|C_{12}\right|^{2}=0.25\left(2 g_{0}^{2}-\bar{\Delta}\right) g_{0}^{2}, \quad\left|C_{11}\right|^{2}=0.25\left(2 g_{0}^{2}+\bar{\Delta}\right) g_{0}^{2},
$$

где $\bar{\Delta}=N^{-1} \sum_{\vec{k}} \Delta(\vec{k})$. Тогда минимальная энергия оказывается равной

$$
\langle d, \psi|\widehat{H}| d, \psi\rangle_{\min }=\sum_{\vec{k}}\left[E_{1}(\vec{k})-\frac{2 g_{0}^{2}-\bar{\Delta}}{8 g_{0}^{2}}\right] .
$$

Параметр деформации, соответствующий минимуму энергии (38),

$$
\left|d_{0}\right|=\sqrt{\frac{N}{8 \omega(0) g_{0}^{2}}} \sqrt{4 g_{0}^{4}-\bar{\Delta}^{2}} .
$$

11. Условием реализации структурной перестройки решетки в случае однородной деформации является условие сильной связи

$$
2 g_{0}^{2}>\bar{\Delta}
$$

при котором квадрат модуля $\left|C_{12}\right|^{2}$ действительно оказывается положительным. Это условие получено выше (см. п. 4) и в работе [11] по межзонной теории сегнетоэлектрических переходов со "смягчением" одной моды колебаний. Но оно имеет вид $4 g_{0}^{2}>\bar{\Delta}$ (коэффициент 4 вместо 2), так как в [11] считается, что и в валентной зоне, и в зоне проводимости могут существовать электроны с двумя ориентациями спина. Мы же для простоты учитывали только одну из этих возможностей. В случае [11] при двух возможных ориентациях спина в соотношениях (37), (38) величина $2 g_{0}^{2}$ заменяется на $4 g_{0}^{2}$, а в соотношении (39) $4 g_{0}^{4}$ - на $16 g_{0}^{4}$. Таким образом, метод варьирования состояний электронной и фононной подсистем при сильном межзонном электрон-фононном взаимодействии позволяет установить связь между деформацией решетки при $T \rightarrow 0 \mathrm{~K}$, возникающей в результате фазового перехода, и изменением функции распределения электронов в элементарной ячейке за счет добавления 
состояний зоны проводимости к состояниям валентных электронов. Полученный критерий сильной связи совпадает с критерием обращения в нуль частоты "мягкой" моды [11] при $T=T_{\mathrm{c}}$, где $T_{\mathrm{c}}$ - температура фазового перехода второго рода (переход типа смещения). Согласно (14) $T_{\mathrm{c}} \approx 4 g_{0}^{2}-\bar{\Delta}$. Это соотношение позволяет установить, что при $\bar{\Delta} \approx 4$ эВ (для кристаллов с широкой запрещенной зоной) и $T_{\mathrm{c}} \approx 400 \mathrm{~K}$ (как, например, у $\left.\mathrm{SbSI}\right) g_{0}^{2} \approx 1.01$ эВ. В рассмотренной спиновой ситуации $2 g_{0}^{2}-\bar{\Delta} \approx 0.01$ эВ для кристалла SbSI. Следовательно, каждый подуровень валентной зоны согласно (38) смещается вниз на величину

$$
\delta E \equiv \frac{\left(2 g_{0}^{2}-\bar{\Delta}\right)^{2}}{8 g_{0}^{2}}=\frac{T_{\mathrm{c}}^{2}}{4 \bar{\Delta}}, \quad \delta E=10^{-4} \frac{\bar{\Delta}}{4} \approx 10^{-4}{ }_{\ni} \mathrm{B} .
$$

Можно говорить, что при завершенной деформации кристалла при $T \rightarrow 0 \mathrm{~K}$ его энергия за счет взаимодействия электронов с деформацией по нормальной координате "мягкой" моды понизится на величину порядка $10^{-4}$ эВ в расчете на одну ячейку кристалла.

12. Очевидно, что температура $T_{\mathrm{c}}>0$ возможна только для тех мод поперечных колебаний среды, для которых

$$
4 g_{0}^{2}(q)>\bar{\Delta}(q)
$$

Учитывая тот факт, что даже в кристаллах миллиметровых размеров набор волновых векторов фононных мод является практически непрерывным, можно быть уверенным, что если неравенство (42) выполняется для некоторой одной моды, то оно выполняется и для достаточно большого множества близких к ней нестабильных мод. Для тех мод, для которых неравенство (42) превращается в равенство, критическая температура $T_{\mathrm{c}}=0 \mathrm{~K}$. Таким образом, можно полагать, что в интервале температур $0 \mathrm{~K} \div T_{c_{\max }}$ произойдет цепочка переходов, связанных с деформацией кристалла по нормальным координатам всех мод указанного множества. В результате оказывается нарушенной трансляционная симметрия кристалла. Очевидно, что при $T \rightarrow 0 \mathrm{~K}$ сформируется доменная структура с размером домена, определяемым шириной области нестабильных мод в зоне Бриллюэна. На размер доменов, конечно, влияет и степень несовершенства кристаллов.

Иначе говоря, рассматриваемая модель фазовых переходов под влиянием межзонного электрон-фононного взаимодействия описывает размытый фазовый переход второго рода. Ширина области размывания зависит от того, в какой области температур происходит деформация по нормальным координатам большинства нестабильных мод. Поскольку модель с гамильтонианом (1) построена на основе предположения о независимости деформаций по нормальным координатам различных мод, то ее выводы не будут справедливы для веществ, в которых моды взаимодействуют между собой непосредственно или через моды других ветвей колебаний кристаллической решетки. При наличии взаимодействия между модами, например стрикции, деформация по нормальным координатам некоторого множества мод может происходить посредством одного скачка без обращения в нуль частот всех этих мод, т.е. в результате фазового перехода второго рода. 


\section{Список литературы}

[1] Б.А. Струков, А.П. Леванюк, Физические основы сегнетоэлектрических явлений в кристаллах, Наука, М., 1983.

[2] А. П. Леванюк, А. Р. Погосян, Е. М. Уюкин, ДАН СССР, 256:1 (1961), 60.

[3] Л. Д. Ландау, Е. М. Лифшиц, Теоретическая физика Т. V. Статистическая физика, Наука, М., 1976.

[4] В. Л. Гинзбург, ФTT, 2:9 (1960), 2031-2134.

[5] L. Onsager, Phys. Rev., 65:3-4 (1944), 117-149.

[6] А.З. Паташинский, В.Л. Покровский, Флуктуационная теория фазовых переходов, Наука, М., 1975.

[7] В. И. Алтухов, Б. А. Струков, "Критическое рассеяние носителей и теории аномального теплового и электрического сопротивления в сегнетоэлектриках", Сб. тезисов докл. XVI Всероссийской конферениии по физике сегнетоэлектриков (Тверь, 17-21 сентября 2002 г.), Тверь, 2002, 62.

[8] В. М. Фридкин, Сегнетоэлектрики-полупроводники, Наука, М., 1976.

[9] Я. Г. Гиршберг, В. И. Тамарченко, ФТТ, 18:4 (1976), 1066-1072.

[10] E. V. Bursian, Ya. G. Girschberg, V. I. Tamarchenko, Ferroelectrics, 18 (1978), 39-43.

[11] Э. В. Бурсиан, Я. Г. Гиршберг, Когерентные эффекты в сегнетоэлектриках, Прометей, М., 1989.

[12] А. А. Абрикосов, Л. П. Горьков, И. Е. Дзялошинский, Методы квантовой теории поля в статистической физике, Физматгиз, М., 1962.

[13] Э. Н. Мясников, А. Э. Мясникова, З. П. Мастропас, ЖЭТФ, 129:3 (2006), 548-565.

Поступила в редакцию 3.01.2008 